Research Article

\title{
Synchronization in Coupled Multistable Systems with Hidden Attractors
}

\author{
Gokul PM and Tomasz Kapitaniak \\ Division of Dynamics, Lodz University of Technology, Stefanowskiego 1/15, 90-457 Lodz, Poland \\ Correspondence should be addressed to Gokul P M; gokulnappu@gmail.com
}

Received 9 December 2016; Revised 17 March 2017; Accepted 20 March 2017; Published 19 April 2017

Academic Editor: Jesus M. Munoz-Pacheco

Copyright (C) 2017 Gokul P M and Tomasz Kapitaniak. This is an open access article distributed under the Creative Commons Attribution License, which permits unrestricted use, distribution, and reproduction in any medium, provided the original work is properly cited.

In this paper, we study the results of coupling multistable systems which have hidden attractors with each other. Three modified Sprott systems were coupled and their synchronization was observed. The final state of the synchronized system changes with the change in the coupling strength. This was seen for two different types of coupling, one with a single variable and the other with two system variables.

\section{Introduction}

Synchronization of dynamical systems has become a field of intense interest and hence extensive study in the last decades [1-8]. Just the dynamical systems alone have been known to exhibit a wide variety of complex behavior.

The reason why synchronization of coupled systems is gaining interest is the huge real life applications that collective behavior [9-11] of such systems has [12-15]. From social behavioral analysis to biological models like neural networks, we have seen the importance and hence the justified interest in the study of this phenomenon increasing.

Since the introduction of the concept of synchronization by Pecora and Carroll [1], there have been a lot of different approaches and therefore types of synchronization observed and documented, like the complete synchronization, phase synchronization [16], lag synchronization [17], and so forth. In our work, we will be studying complete synchronization of the system presented.

Now looking at behaviors of dynamical systems, a recent concept that is becoming increasingly interesting is the concept of multistability and hidden attractors [18-22]. Multistable systems are those dynamical systems that have more than one equilibrium point for a given set of parameters. This results in the final state of the system being very sensitive to the initial conditions and also to perturbations.
Another interesting concept is the hidden attractor. Hidden attractors are attractors whose basins do not intersect with small neighborhoods of equilibria. This results in needing special methods to find them as standard methods become insufficient. These kinds of attractors were first observed by Yang et al. [23] who, when studying a system with two stable equilibrium points, found that the same system exhibited chaotic behavior. These attractors were later called hidden attractors by Leonov et al. [24]. There have been a lot of further studies in this field, since the systems show multistability, and have resulted in hidden attractors being shown using electronic circuits by Saha et al. $[25,26]$. This shows actual physical realization of the theoretical concept. Furthermore, the system introduced by Sprott [27-29], and later studied in a more generalized way by Wang et al. [30, 31], has shown similar behavior. This is important to our case as it will be the base system we will be using in our studies.

In our study, we have decided to take a multistable system with hidden attractors [30] and study the complete synchronization of three such systems coupled simply. While there have been studies done on seeing the transition between two attractors in a multistable system [32] and even on generalized synchronization of such attractor [33], we will be observing the complete synchronization of our system and how multistability of the system affects the observed synchronization. 


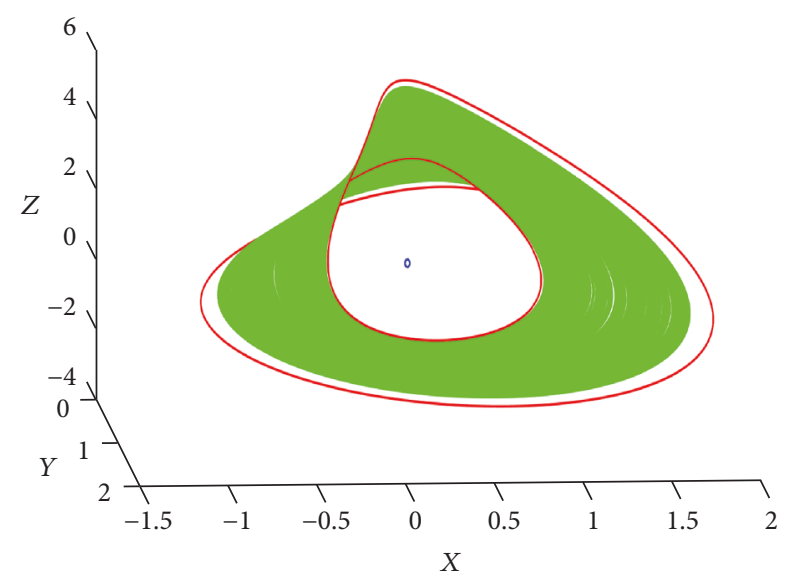

Figure 1: Phase space plot for the uncoupled system (1) where the blue point gives the stable equilibrium point, the green line gives the strange attractor which is chaotic, and the red line denotes the hidden attractor which is in period-three limit cycle.

\section{System Model}

In this work, we study the different synchronization of one such system proposed by Wang and Chen [30], which in itself was a more generalized version of the one proposed by Sprott [27-29]. Wang-Chen system is described by

$$
\begin{aligned}
& \frac{d x}{d t}=y z+a, \\
& \frac{d y}{d t}=x^{2}-y, \\
& \frac{d z}{d t}=1-4 x
\end{aligned}
$$

where $x, y$, and $z$ are three state variables, while $a$ is the parameter.

This system has a hidden attractor and shows multistability and the parameter $a$ is chosen in such a way that the final state is very sensitive to the initial condition. There are three attractors in the system to begin with: a point attractor, a strange attractor, and a hidden attractor. In our system, we have chosen $a=0.01$, so that the stable attractor converges to a point, the hidden attractor is in period-three state, and the strange attractor is chaotic as illustrated in Figure 1.

\section{Single Variable Coupling}

There are a variety of ways in which the system mentioned can be coupled. We have chosen a simple unidirectional coupling but with all $x, y$, and $z$ being coupled one at a time. The figures below show the results, where the initial condition of the individual systems would have all taken them to the hidden attractor had there been no coupling. The plot shows how the difference, $\Delta_{i j}$ changes with the coupling strength, where

$$
\Delta_{i j}=\frac{1}{N} \sum_{l=1}^{N} \sqrt{\left(x_{i}^{l}-x_{j}^{l}\right)^{2}+\left(y_{i}^{l}-y_{j}^{l}\right)^{2}+\left(z_{i}^{l}-z_{j}^{l}\right)^{2}}
$$

with $i, j=1,2,3$, and $l=1,2, \ldots, N$. Here $l$ denotes the last few iterations over which we have taken the mean. In our work, the value of $\Delta_{i j}$ is corresponding to the synchronization error. For example, when the synchronization occurs the value of $\Delta_{i j}<0 \pm 0.0001$.

It can be seen from Figure 2 that there are multiple regions of synchronization. When these regions were studied individually, they showed that the final synchronized state was not the same at all these points. It was also seen that, at every point of synchronization, there is a complete synchronization of all the variables with every other variable.

Let us take the case of unidirectional $x$-coupling alone, whose equation is

$$
\begin{aligned}
& \dot{x_{1}}=y_{1} z_{1}+a+k\left(x_{2}-x_{1}\right), \\
& \dot{y}_{1}=x_{1}^{2}-y_{1}, \\
& \dot{z}_{1}=1-4 x_{1}, \\
& \dot{x_{2}}=y_{2} z_{2}+a+k\left(x_{3}-x_{2}\right), \\
& \dot{y}_{2}=x_{2}^{2}-y_{2}, \\
& \dot{z_{2}}=1-4 x_{2}, \\
& \dot{x_{3}}=y_{3} z_{3}+a+k\left(x_{1}-x_{3}\right), \\
& \dot{y_{3}}=x_{3}^{2}-y_{3}, \\
& \dot{z_{3}}=1-4 x_{3} .
\end{aligned}
$$

After studying the individual cases, it was seen that the final region of synchronization changes from one attractor to another at the different synchronization regions (see Figure 3). Since it was seen that all variables, that is, $x, y$ and $z$, synchronize whenever a single one synchronizes, we can just study the final state of a single system and conclude that all the systems are in this same state. The synchronized states start with the system being in the fixed point attractor $(k=0.15)$ which then changes to the period-three attractor (from $k=0.32$ ) and finally to the chaotic attractor $(k=0.36)$, where it stays for all other increases.

Now, when we try the same using $y$-coupling, synchronization only occurs in a small region. Here both the regions of synchronization are where the system finally reaches the fixed point. No other regions were observed. When repeated for the $z$-coupling, the result was that there was a large region of synchronization like in the case of $x$-coupling, but the final synchronized state was always in the fixed point attractor, like in the $y$-coupling.

\section{Two-Variable Coupling}

Next we tried the same unidirectional coupling, but now with two variables. For example, the equations for $x y$-coupling looks like

$$
\begin{aligned}
& \dot{x_{1}}=y_{1} z_{1}+a+k\left(x_{2}-x_{1}\right), \\
& \dot{y_{1}}=x_{1}^{2}-y_{1}+k\left(y_{2}-y_{1}\right),
\end{aligned}
$$




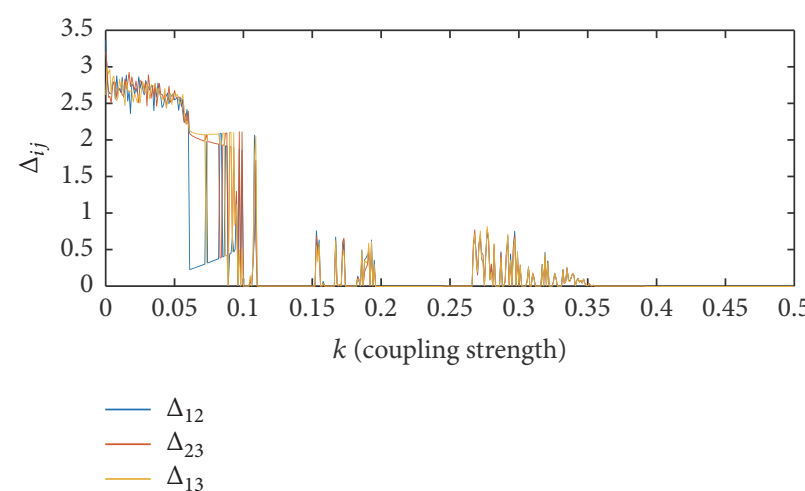

(a)

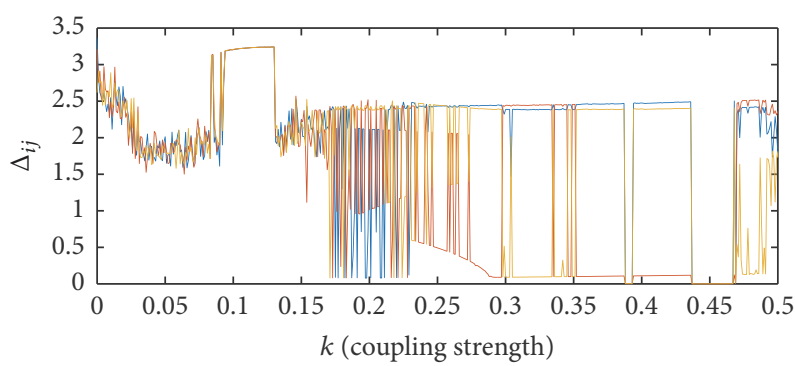

$\Delta_{12}$

$-\Delta_{23}$

(b)

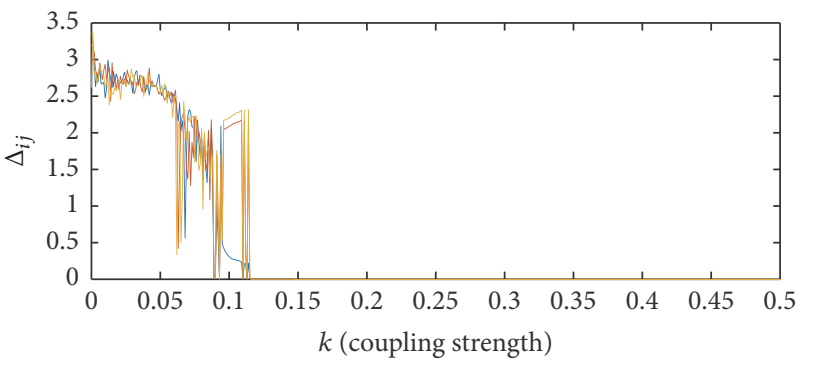

$-\Delta_{12}$
$-\Delta_{23}$
$-\Delta_{13}$

(c)

Figure 2: Change of the absolute difference between the variables $\Delta$ after a long period of time with increasing coupling strength, with (a), (b), and (c) being the plots for $x$-, $y$-, and $z$-coupling, respectively.

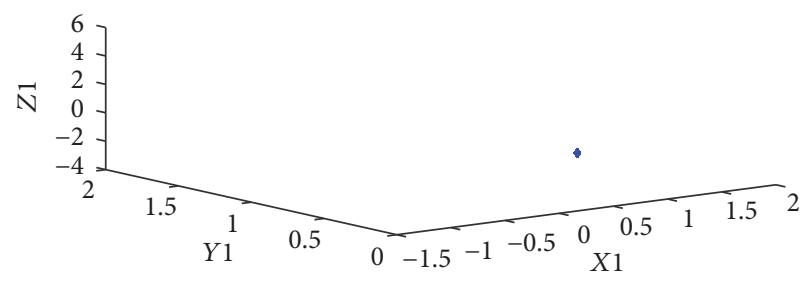

(a)

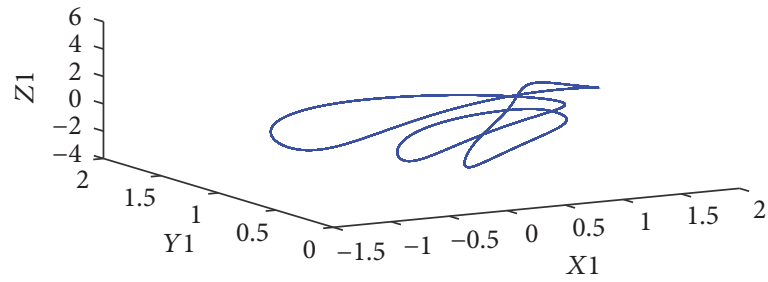

(b)

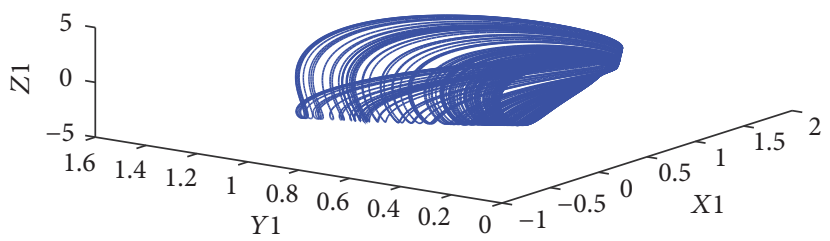

(c)

Figure 3: The final state of the variables of the first system at different coupling strength for $x$-coupling only. Figures (a), (b), and (c) show the phase space at $k=0.15,0.32$, and 0.36 , respectively.

$$
\begin{aligned}
& \dot{z_{1}}=1-4 x_{1}, \\
& \dot{x_{2}}=y_{2} z_{2}+a+k\left(x_{3}-x_{2}\right), \\
& \dot{y}_{2}=x_{2}^{2}-y_{2}+k\left(y_{3}-y_{2}\right), \\
& \dot{z_{2}}=1-4 x_{2},
\end{aligned}
$$$$
\begin{aligned}
& \dot{x_{3}}=y_{3} z_{3}+a+k\left(x_{1}-x_{3}\right), \\
& \dot{y}_{3}=x_{3}^{2}-y_{3}+k\left(y_{1}-y_{3}\right), \\
& \dot{z_{3}}=1-4 x_{3} .
\end{aligned}
$$ 


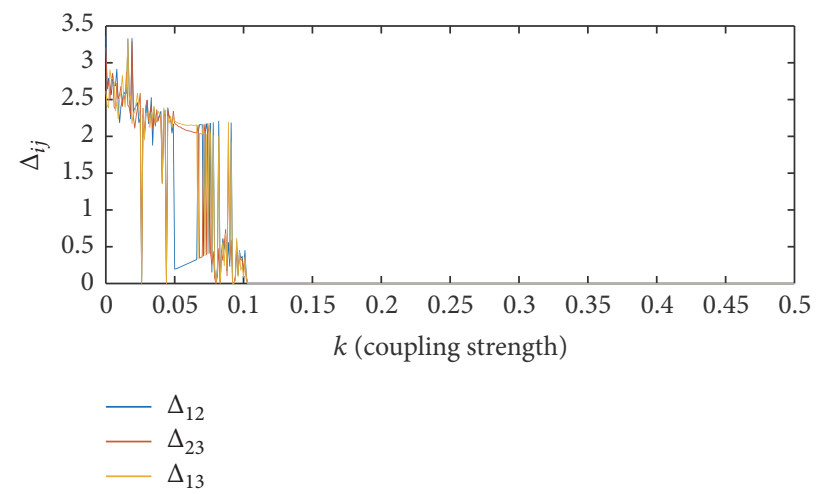

(a)

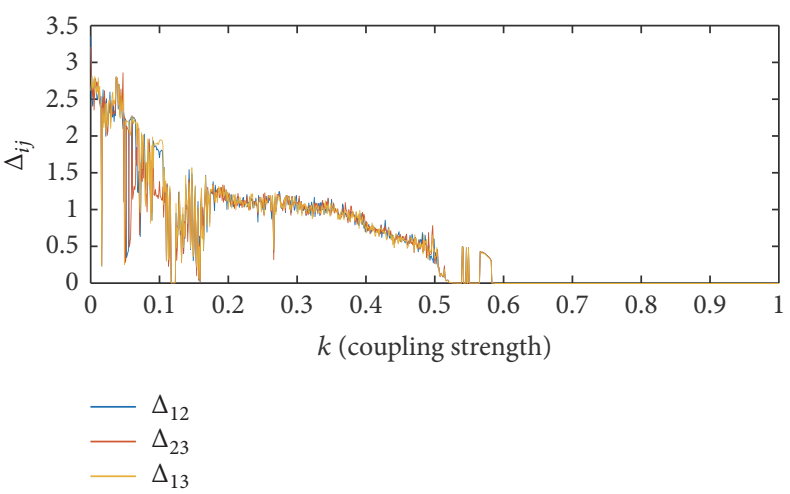

(b)

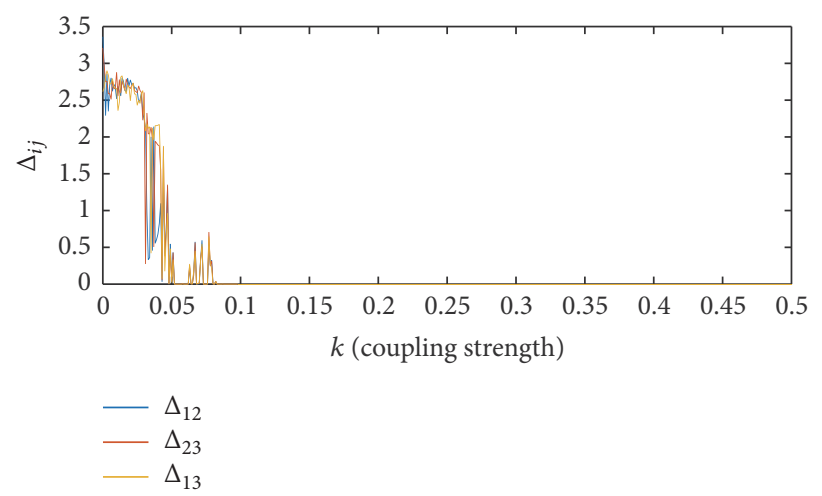

(c)

FIGURE 4: Change of the absolute difference between the variables $\Delta$ after a long period of time with increasing coupling strength, with (a), (b), and (c) being the plots for $x y$-, $y z$-, and $x z$ - coupling, respectively.

Many regions of synchronization were observed separated by a desynchronized region. Like the previous case, we expected these regions to show different behavior, which is true, though the order is different, with the system going from point attractor to chaotic to period-three attractor as seen in Figure 4 . In the case of $x y$-coupling and $x z$-coupling, that was the final synchronized state, but we saw that, for $y z$ coupling, the final synchronized state undergoes one more shift into the chaotic attractor, although this time it is without the separation of a desynchronized region. This can be seen from Figures 5 and 6, respectively.

\section{Conclusion}

In the model studied, we observed synchronization of three systems that were introduced by Sprott et al., in the case of two types of unidirectional coupling, using one variable and then two. We studied the case where the individual systems were in period-three hidden attractor. It was seen that, depending on the strength of coupling between the systems, not only was the synchronization affected, but also the final synchronized state was affected. While the one-variable coupling that gave the most interesting result was for $x$-coupling, where the final state shifted from point attractor to the chaotic one via the period-three attractor, the two-variable coupling gave for $x y$-coupling and $x z$-coupling the same phenomenon of transition from point attractor to chaotic attractor to periodthree attractor, whereas the $y z$-coupling made one more final transition to the chaotic attractor.

Our work emphasizes this observed synchronization, which, depending on the coupling strength alone, changes its final state from a stable equilibrium point to the strange and hidden attractors in that order or vice versa depending on the type of coupling. This change is interesting and we attribute this effect to the multistability of the system which made it very sensitive to perturbations.

Since the uncoupled system is very sensitive to the initial conditions, we tried to repeat the whole process with different initial condition, where the initial uncoupled system was in different attractors, including cases where each system was in a different attractor. Similar results were observed, where the system started from the point attractor and then moved to the periodic or chaotic attractor and then again moved on to a different attractor.

\section{Conflicts of Interest}

The authors declare that they have no conflicts of interest. 


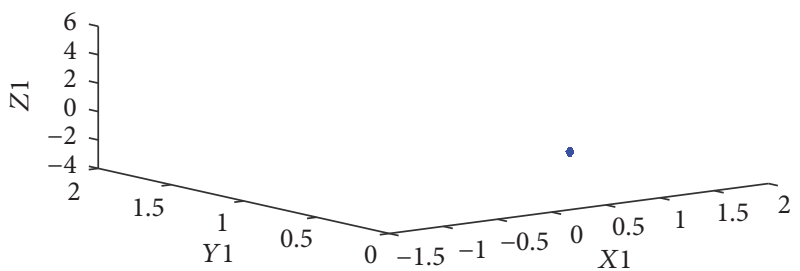

(a)

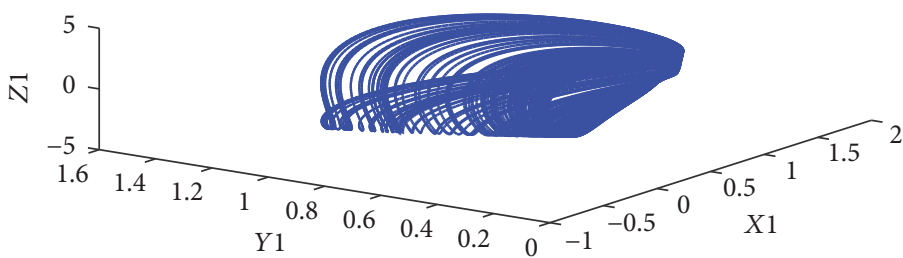

(b)

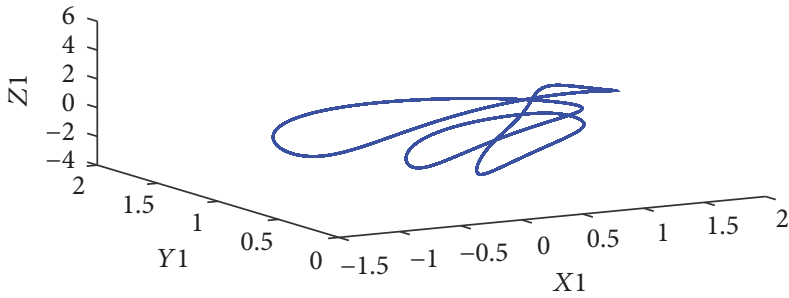

(c)

Figure 5: The final state of the variables of the first system at different coupling strength for $x y$-coupling. Figures (a), (b), and (c) show the phase space plot of one system at $k=0.08,0.11$, and 0.12 , respectively.

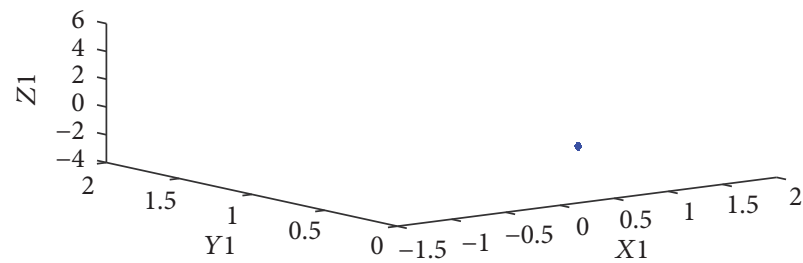

(a)

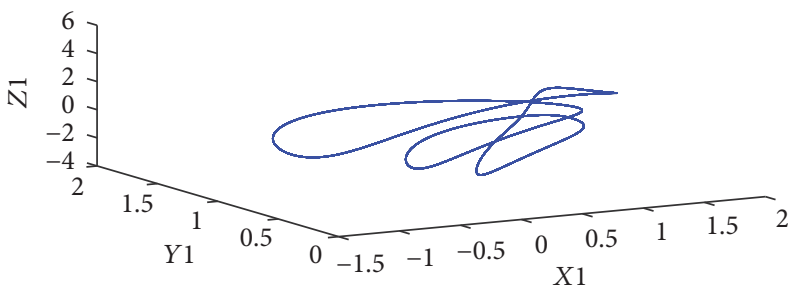

(c)

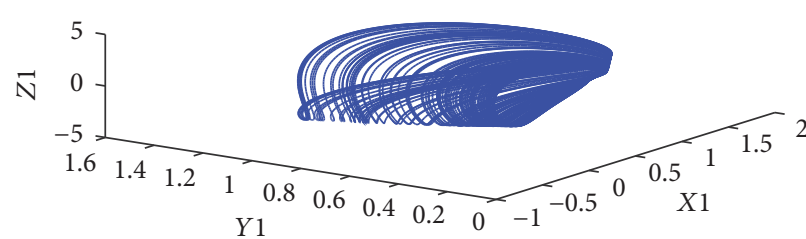

(b)

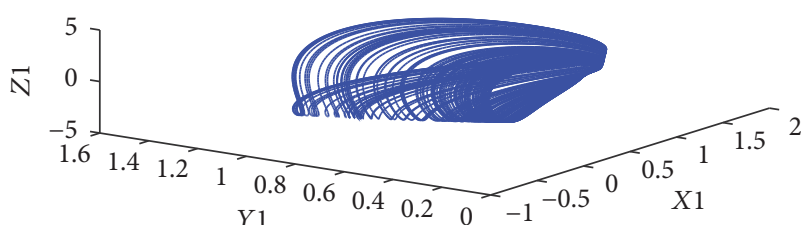

(d)

Figure 6: The final state of the variables of the first system at different coupling strength for $y z$-coupling only. Figures (a), (b), (c), and (d) show the phase space plot of one system at $k=0.12,0.53,0.849$, and 2.81 , respectively.

\section{Acknowledgments}

This work has been supported by the Polish National Science Centre, MAESTRO Programme, Project no. 2013/08/A/ ST8/00/780.

\section{References}

[1] L. M. Pecora and T. L. Carroll, "Synchronization in chaotic systems," Physical Review Letters, vol. 64, no. 8, pp. 821-824, 1990.

[2] S. Yanchuk and T. Kapitaniak, "Chaos-hyperchaos transition in coupled Rössler systems," Physics Letters, A, vol. 290, no. 3-4, pp. 139-144, 2001.

[3] S. Yanchuk and T. Kapitaniak, "Symmetry-increasing bifurcation as a predictor of a chaos-hyperchaos transition in coupled systems," Physical Review E, vol. 64, no. 5, Article ID 056235, 2001.

[4] M. Kapitaniak, K. Czolczynski, P. Perlikowski, A. Stefanski, and T. Kapitaniak, "Synchronization of clocks," Physics Reports, vol. 517, no. 1-2, pp. 1-69, 2012.

[5] M. Kapitaniak, K. Czolczynski, P. Perlikowski, A. Stefanski, and T. Kapitaniak, "Synchronous states of slowly rotating pendula," Physics Reports, vol. 541, no. 1, pp. 1-44, 2014.

[6] M. Kapitaniak, P. Brzeski, K. Czolczynski, P. Perlikowski, A. Stefanski, and T. Kapitaniak, "Synchronization thresholds of coupled self-excited nonidentical pendula suspended on the vertically displacing beam," Progress of Theoretical Physics, vol. 128, no. 6, pp. 1141-1173, 2012.

[7] P. Kuzma, M. Kapitaniak, and T. Kapitaniak, "Coupling multistable systems: uncertainty due to the initial positions in the 
attractors," Journal of Theoretical and Applied Mechanics, vol. 52, no. 1, p. 281, 2014.

[8] M. Kapitaniak, M. Lazarek, M. Nielaczny, K. Czolczynski, P. Perlikowski, and T. Kapitaniak, "Synchronization extends the life time of the desired behavior of globally coupled systems," Scientific Reports, vol. 4, article 4391, 2014.

[9] A. S. Pikovsky, M. G. Rosenblum, and J. Kurths, Synchronization: A Universal Concept in Nonlinear Sciences, Cambridge University Press, Cambridge, UK, 2002.

[10] S. Boccaletti, J. Kurths, G. Osipov, D. L. Valladares, and C. S. Zhou, "The synchronization of chaotic systems," Physics Report, vol. 366, no. 1-2, pp. 1-101, 2002.

[11] L. Kocarev and U. Parlitz, "Generalized synchronization, predictability, and equivalence of unidirectionally coupled dynamical systems," Physical Review Letters, vol. 76, no. 11, pp. 18161819, 1996.

[12] B. F. Kuntsevich and A. N. Pisarchik, "Synchronization effects in a dual-wavelength class-B laser with modulated losses," Physical Review E, vol. 64, no. 4, Article ID 046221, 2001.

[13] M. I. Rabinovich, P. Varona, A. I. Selverston, and H. D. I. Abarbanel, "Dynamical principles in neuroscience," Reviews of Modern Physics, vol. 78, no. 4, Article ID 1213, 2006.

[14] B. Blasius, A. Huppert, and L. Stone, "Complex dynamics and phase synchronization in spatially extended ecological systems," Nature, vol. 399, no. 6734, pp. 354-359, 1999.

[15] D. J. Watts and S. H. Strogatz, "Collective dynamics of "smallworld” networks," Nature, vol. 393, no. 6684, pp. 440-442, 1998.

[16] M. G. Rosenblum, A. S. Pikovsky, and J. Kurths, "Phase synchronization of chaotic oscillators," Physical Review Letters, vol. 76, no. 11, pp. 1804-1807, 1996.

[17] M. G. Rosenblum, A. S. Pikovsky, and J. Kurths, "From phase to lag synchronization in coupled chaotic oscillators," Physical Review Letters, vol. 78, no. 22, pp. 4193-4196, 1997.

[18] Y. Maistrenko, S. Brezetskyi, P. Jaros, R. Levchenko, and T. Kapitaniak, "Smallest chimera states," Physical Review E, vol. 95, Article ID 010203, 2017.

[19] S. Brezetskyi, D. Dudkowski, and T. Kapitaniak, "Rare and hidden attractors in Van der Pol-Duffing oscillators," European Physical Journal: Special Topics, vol. 224, no. 8, pp. 1459-1467, 2015.

[20] A. Chudzik, P. Perlikowski, A. Stefanski, and T. Kapitaniak, "Multistability and rare attractors in van der Pol-Duffing oscillator," International Journal of Bifurcation and Chaos, vol. 21, no. 7, pp. 1907-1912, 2011.

[21] T. Kapitaniak and G. A. Leonov, "Multistability: uncovering hidden attractors," European Physical Journal: Special Topics, vol. 224, no. 8, pp. 1405-1408, 2015.

[22] J. C. Sprott, "Strange attractors with various equilibrium types," The European Physical Journal Special Topics, vol. 224, no. 8, pp. 1409-1419, 2015.

[23] Q. Yang, Z. Wei, and G. Chen, "An unusual 3D autonomous quadratic chaotic system with two stable node-foci," International Journal of Bifurcation and Chaos, vol. 20, no. 4, pp. 10611083, 2010.

[24] G. A. Leonov, N. V. Kuznetsov, and V. I. Vagaitsev, "Hidden attractor in smooth Chua systems," Physica D: Nonlinear Phenomena, vol. 241, no. 18, pp. 1482-1486, 2012.

[25] P. Saha, D. C. Saha, A. Ray, and A. RoyChowdhary, "Multistability in a single system with hidden attractors: theory and experiment," International Journal of Physics, vol. 2, no. 6, pp. 217-225, 2014.
[26] P. Saha, D. C. Saha, A. Ray, and A. R. Chowdhury, "Memristive non-linear system and hidden attractor," European Physical Journal: Special Topics, vol. 224, no. 8, pp. 1563-1574, 2015.

[27] J. C. Sprott, "Automatic generation of strange attractors," Computers and Graphics, vol. 17, no. 3, pp. 325-332, 1993.

[28] J. C. Sprott, "Some simple chaotic flows," Physical Review E, vol. 50, no. 2, pp. R647-R650, 1994.

[29] J. C. Sprott, "Simplest dissipative chaotic flow," Physics Letters. $A$, vol. 228, no. 4-5, pp. 271-274, 1997.

[30] X. Wang and G. Chen, "A chaotic system with only one stable quilibrium point," Communications in Nonlinear Science and Numerical Simulation, vol. 17, Article ID 12641272, pp. 12641272, 2012.

[31] J. C. Sprott, X. Wang, and G. Chen, "Coexistence of point, periodic and strange attractors," International Journal of Bifurcation and Chaos, vol. 23, no. 5, Article ID 1350093, 2013.

[32] A. N. Pisarchik, R. Jaimes-Reátegui, J. R. Villalobos-Salazar, J. H. García-López, and S. Boccaletti, "Synchronization of chaotic systems with coexisting attractors," Physical Review Letters, vol. 96, no. 24, Article ID 244102, 2006.

[33] A. Hu and Z. Xu, "Multi-stable chaotic attractors in generalized synchronization," Communications in Nonlinear Science and Numerical Simulation, vol. 16, no. 8, pp. 3237-3244, 2011. 


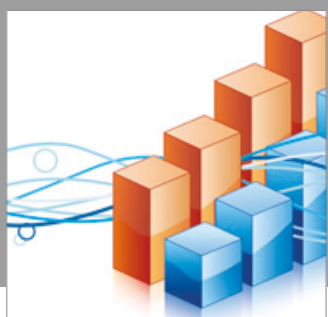

Advances in

Operations Research

vatersals

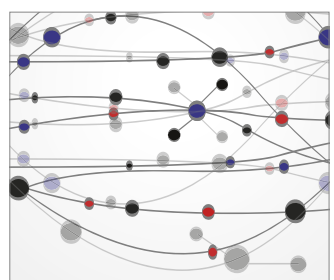

\section{The Scientific} World Journal
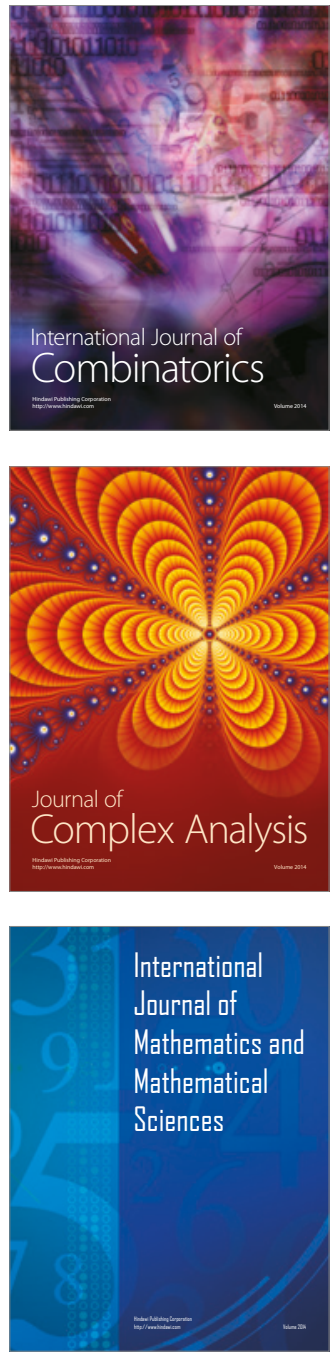
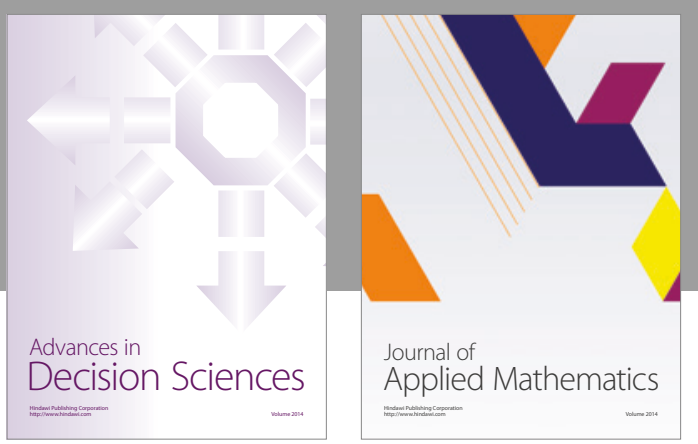

Algebra

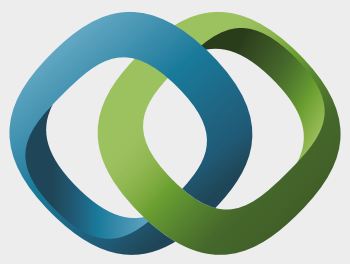

\section{Hindawi}

Submit your manuscripts at

https://www.hindawi.com
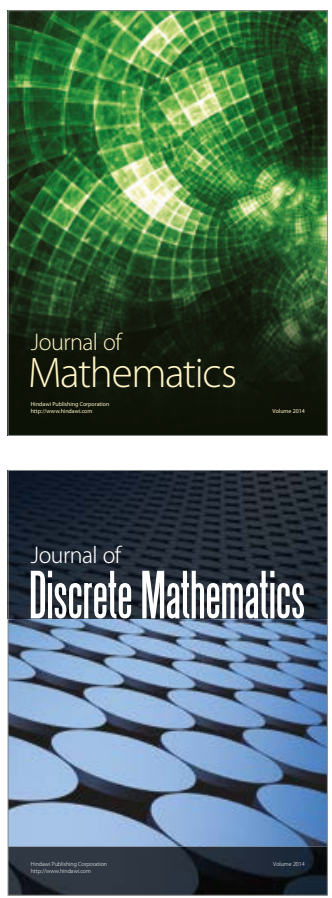

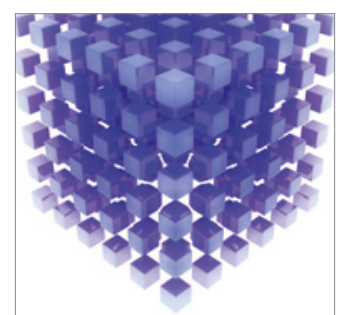

Mathematical Problems in Engineering
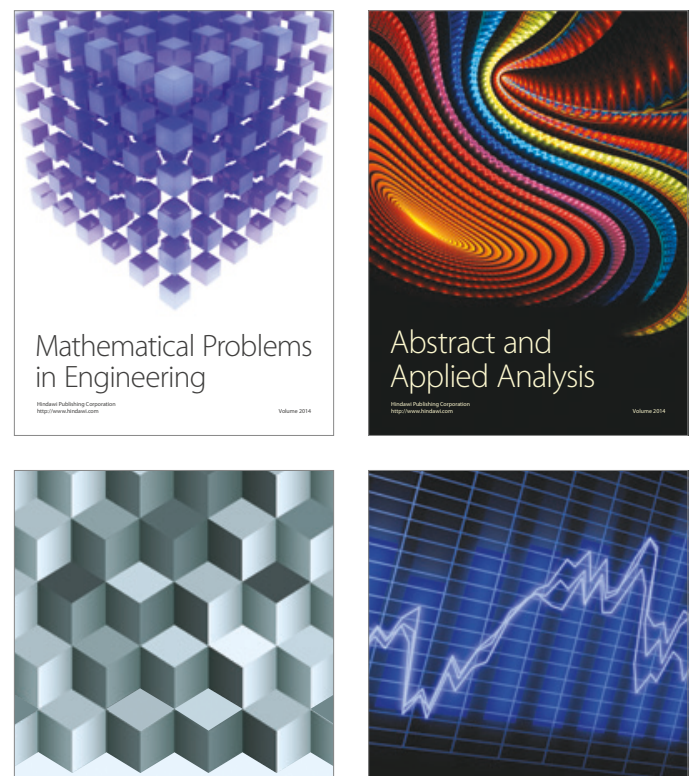

Journal of

Function Spaces

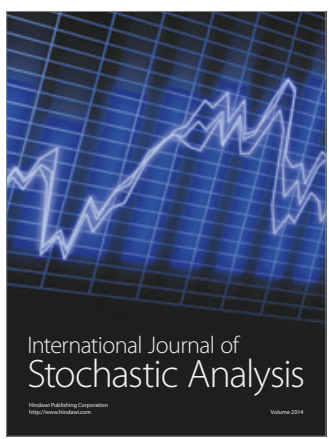

Probability and Statistics
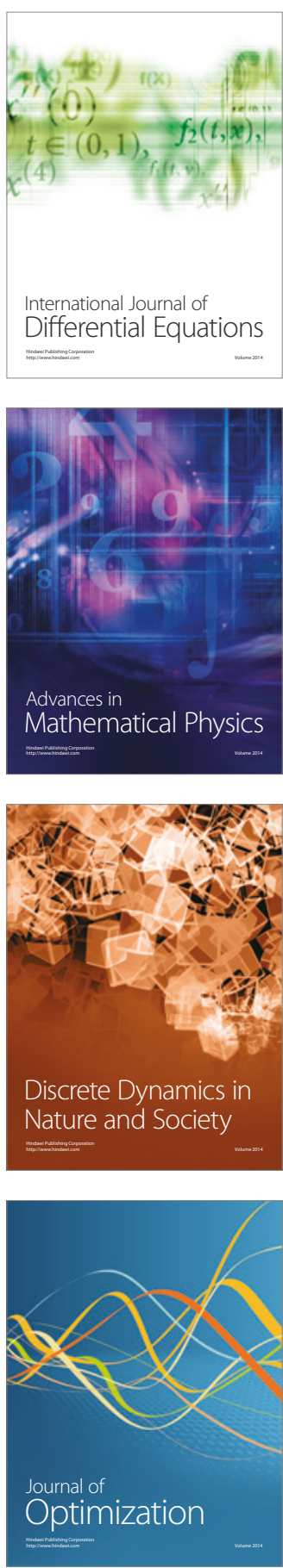